\title{
Utilização de biossurfactante no escoamento de petróleo em campo maduro
}

\author{
Use of biosurfactant in the oil flow in mature field
}

\author{
A. K. de S. N. Soares ${ }^{1}$; R. R. de Souza ${ }^{2 *}$ \\ ${ }^{1}$ Programa de Pós-Graduação em Engenharia Química, Laboratório de Biotecnologia Ambiental (LABAM), \\ Universidade Federal de Sergipe, 49100-000, São Cristóvão-Sergipe, Brasil. Tel. +55 (79) 2105-6549. \\ ${ }^{2}$ Programa de Pós-Graduação em Engenharia Química, Laboratório de Biotecnologia Ambiental (LABAM), \\ Departamento de Engenharia Química, Universidade Federal de Sergipe, 49100-000, São Cristóvão-Sergipe, Brasil. \\ Tel. +55 (79) 2105-6687 \\ *rrsouza@ufs.br \\ (Recebido em 15 de março de 2016; aceito em 08 de maio de 2016)
}

\begin{abstract}
Os biossurfactantes são compostos orgânicos que podem ser utilizados como redutores das altas tensões interfaciais entre o óleo e a água contidos em rochas reservatório, tendo como consequência um aumento nos índices de recuperação de óleo. Com objetivo de avaliar a mobilidade do petróleo em meio poroso foram analisadas as características do meio e dos fluidos, simulando as condições reais de pressão e temperatura na produção de óleo em um poço para produção de biossurfactante e para análise da recuperação do óleo. Como resultado das análises propostas para o biossurfactante em estudo foi determinada a concentração micelar crítica (CMC), com valor de $50 \mathrm{mg} / \mathrm{L}$, apresentando estabilidade frente a elevadas temperaturas e altas concentrações salinas. O meio poroso estudado apresentou uma porosidade em torno de $39,78 \%$ e uma permeabilidade de $124,37 \mathrm{mD}$. Na análise de recuperação de petróleo os resultados mostraram um fator de recuperação parcial de 30,66 \% e o fator de recuperação total de 50,47 \% com a injeção de biossurfactante. Os resultados obtidos permitem concluir que a utilização do biossurfactante produzido tendo como substrato a manipueira e o micro-organismo Bacillus subtilis pode ser usado no processo de recuperação avançada de petróleo melhorada por micro-organismos - MEOR.
\end{abstract}

Palavras-chave: biossurfactante, petróleo, campo maduro.

The biosurfactants are organic compounds which can be used as reducing the high interfacial tension between the oil and water contained in reservoir rocks, resulting in an increase in oil recovery rates. In order to evaluate the petroleum mobility in porous media, the characteristics of the medium and fluid were analyzed by simulating the actual conditions of pressure and temperature in the production of oil into a well to biosurfactant production and for analysis of oil recovery. As a result of analysis proposed for biosurfactant under study it was determined at the critical micelle concentration (CMC), with a value of $50 \mathrm{mg} / \mathrm{L}$, showing stability against high temperature and high salt concentrations. The porous medium studied had a porosity of about $39.78 \%$ and $124.37 \mathrm{mD}$ permeability. In the oil recovery analysis results showed a partial recovery factor of $30.66 \%$ and the total recovery factor of $50.47 \%$ with the injection of biosurfactant. The results showed that the use of biosurfactant produced as substrate manipueira and the microorganism Bacillus subtilis can be used in advanced recovery process oil improved by microorganisms - MEOR.

Keywords: biosurfactant, oil, mature field.

\section{INTRODUÇÃO}

A extração do petróleo é realizada nos reservatórios por diferentes métodos de recuperação, a saber: Método primário - onde o poço tem pressão suficiente para elevar o óleo contido na rocha; Método secundário - onde é necessário a injeção de outro fluido, como por exemplo água, fazendo com que a pressão no reservatório aumente e o óleo seja recuperado; e Método terciário - onde estão inclusos o metódo de recuperação avançada de petróleo (Enhanced Oil Recovery - EOR) e o método de recuperação avançada de petróleo melhorada por micro-organismos (Microbially Enhanced Oil Recovery - MEOR) [1].

As novas descobertas de reservas de petróleo e a grande demanda desta matéria prima em escala mundial tem levado a estudos na melhoria dos métódos de recuperação existentes e/ou a criação de novos métodos com o objetivo de aumentar a capacidade produtiva de cada poço. Assim, uma das 
principais alternativas utilizadas na recuperação do petróleo, tem sido a utilização de biossurfactante em substituição ao surfactantes químicos, pois trata-se de um produto de baixo impacto ambiental devido a sua biodegradabilidade e melhor fator de recuperção quando comparado com os produtos convencionais $[2,3]$.

A utilização do biossurfactante deve-se a redução na tensão interfacial que gera um aumento na mobilidade do petróleo no meio poroso, fazendo com que a recuperação do petróleo seja melhorada [1]. A ação do biossurfactante pode aumentar em torno de $25 \%$ o fator de recuperação do petróleo, mesmo nas condições extremas de temperatura, $\mathrm{pH}$ e salinidade que são caracteristicas inerentes aos poços produtores de petróleo [4]. As propriedades emulsificantes e estabilidade do biossurfactante produzido por Bacillus subtilis tendo como substrato a manipueira foram determinadas e mostra-se semelhente aos sufactantes químicos $[2,3]$.

O presente trabalho visa à avaliação da mobilidade do óleo num meio poroso, através da utilização do método de recuperação terciário MEOR, utilizando o biosurfactante do tipo surfactina, produzido por Bacillus subtilis, tendo como substrato o resíduo agroindustrial manipueira.

\section{MATERIAL E MÉTODOS}

\subsection{Micro-organismo}

O micro-organismo utilizado foi a cepa Bacillus subtilis denominada ICF-PC previamente isolada de borra de petróleo e selecionada como produtora de surfactina em meio contendo a manipueira, esta cepa integra a coleção de culturas do Laboratório de Biotecnologia Ambiental (LABAM) do Departamento de Engenharia Química da Universidade Federal de Sergipe.

\subsection{Inóculo}

A preparação do inóculo foi seguida a metodologia, esta etapa visa a ativação e renovação das células microbianas [5].

\subsection{Fermentação}

A preparação do caldo fermentativo seguiu as mesmas condições definidas [5] e ajustada apenas na utilização da manipueira que foi a $70 \%$ e o pH ajustado para 6,0 com solução de $\mathrm{NaOH}$. O caldo fermentado livre de células contendo o biossurfactante foi submetido às análises e aplicações deste trabalho.

\subsection{Recuperação do biossurfactante}

$\mathrm{O}$ caldo fermentado contendo o biossurfactante extracelular foi acidificado até pH 2,0 com $\mathrm{H}_{2} \mathrm{SO}_{4}$ concentrado e deixado por 12 horas a $4{ }^{\circ} \mathrm{C}$ para precipitação do bioproduto (biossurfactante). Após, o precipitado formado foi centrifugado e o biossurfactante bruto foi seco até atingir peso constante [5].

\subsection{Determinação da CMC}

A CMC (Concentração Micelar Crítica) do biossurfactante foi determinada utilizando o equipamento Tensiômetro SIGMA modelo KSV 700. Para a realização dos ensaios foi utilizada 0,030 L da solução de biossurfactante para cada análise $\left(26^{\circ} \mathrm{C}\right)$. As concentrações das soluções contendo o biossurfactante variaram de $2 \times 10^{-6} \mathrm{~kg} / \mathrm{L}$ até concentrações com valores de tensão superficial próximos aos da água $(\gamma \mathrm{H} 2 \mathrm{O}=71,892 \mathrm{mN} / \mathrm{m}), 1 \times 10^{-6} \mathrm{~kg} / \mathrm{L}$.

\subsection{Etapas de injeção dos fluidos}

Essa etapa tem por objetivo injetar os fluidos (salmoura, óleo) de forma a saturar e avaliar a recuperação do óleo no meio poroso, com isso determinar a permeabilidade. Todas as etapas deste procedimento seguirão a metodologia [6]. 


\subsection{Determinação da quantidade de óleo recuperado}

A quantidade de óleo recuperado foi realizada coletando amostras em intervalos regulares de tempo, sendo a massa de óleo presente na fase aquosa determinada através do teor de óleos e graxas (TOG) medida no equipamento Horiba oil content Analyzer.

\section{RESULTADOS E DISCUSSÃO}

\subsection{Determinação da CMC}

Os biossurfactante possuem CMC entre 0,001 a $2 \times 10^{-3} \mathrm{~kg} / \mathrm{L}$, neste estudo podemos observar a CMC encontrada foi de $50 \times 10^{-6} \mathrm{~kg} / \mathrm{L}$ sem a presença de meio salino, como mostrado na Figura 1. Este resultado está em consonância com os valores citados na literatura [7, 8] em trabalhos realizados visando o estudo comparativo da produção de biossurfactante produzido por microorganismos isolados de reservatório de petróleo.

Na realização dos experimentos para as demais determinações foi utilizada uma concentração do biossurfactante de $30 \%$ acima da CMC, visando garantir que o biossurfactante encontra-se na concentração para a formação de micelas, logo, o valor da concentração utilizado nos experimentos foi de $65 \times 10^{-6} \mathrm{~kg} / \mathrm{L}$ de biossurfactante.

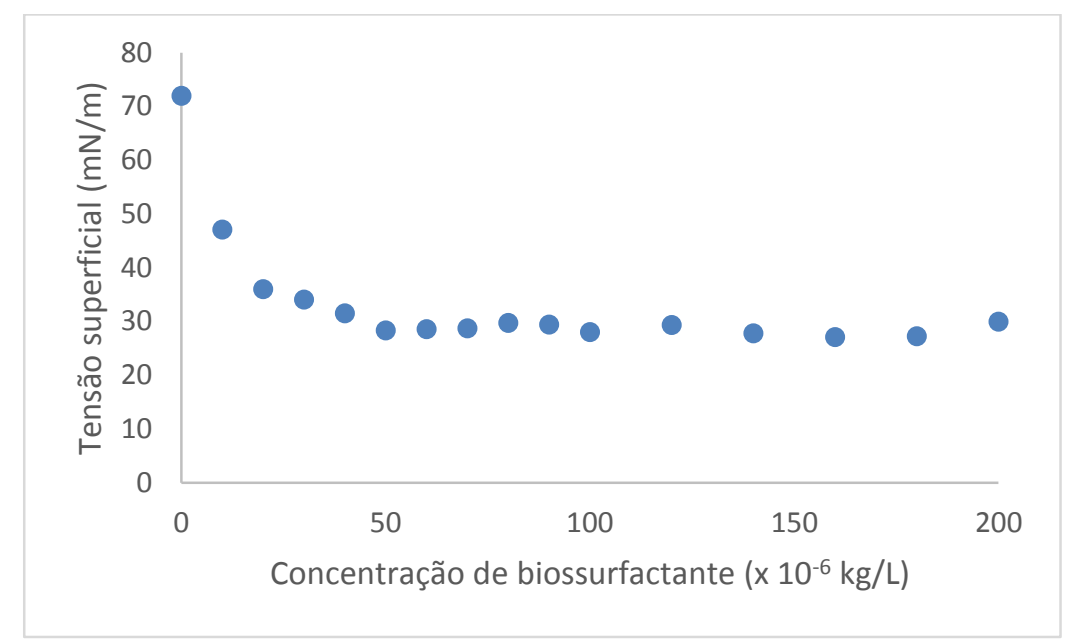

Figura 1: Curva para determinação da CMC

\subsection{Permeabilidade absoluta}

A permeabilidade absoluta foi determinada na etapa de saturação da água, onde existia apenas a água no meio poroso. A Figura 2 representa a permeabilidade da água versus a saturação da mesma.

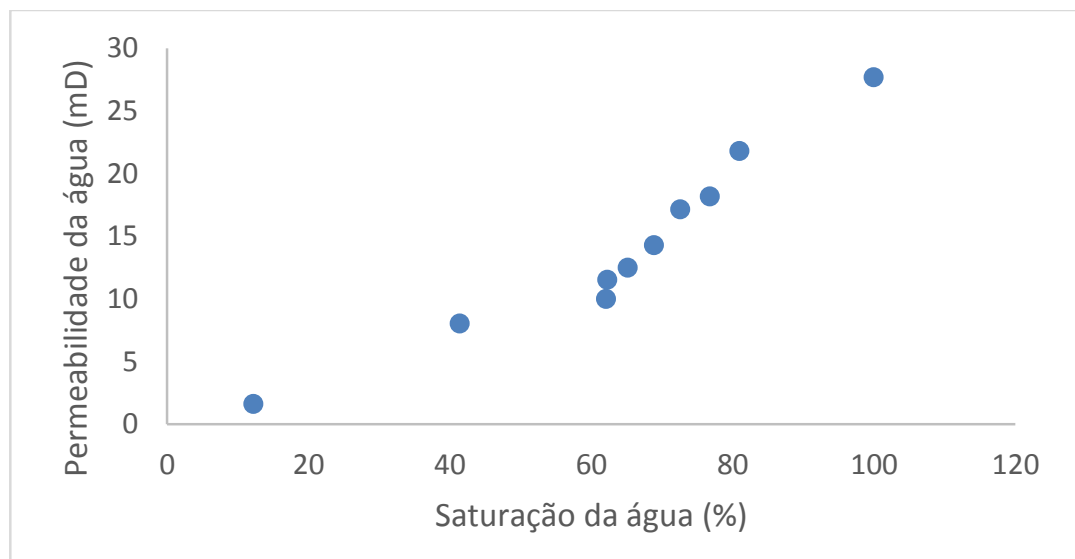

Figura 2: Permeabilidade absoluta para água 
O valor encontrado para permeabilidade do sistema em estudo foi de $27,69 \mathrm{mD}$, o que segundo a classificação [9], esse valor representa uma permeabilidade do meio poroso que permitir o fluxo de fluido com facilidade.

\subsection{Permeabilidade Efetiva ao óleo}

A permeabilidade é uma propriedade que possibilita determinar a capacidade com que cada fluido existente na rocha consegue se mover. A Figura 3 apresenta a permeabilidade efetiva ao óleo em função da saturação da água.

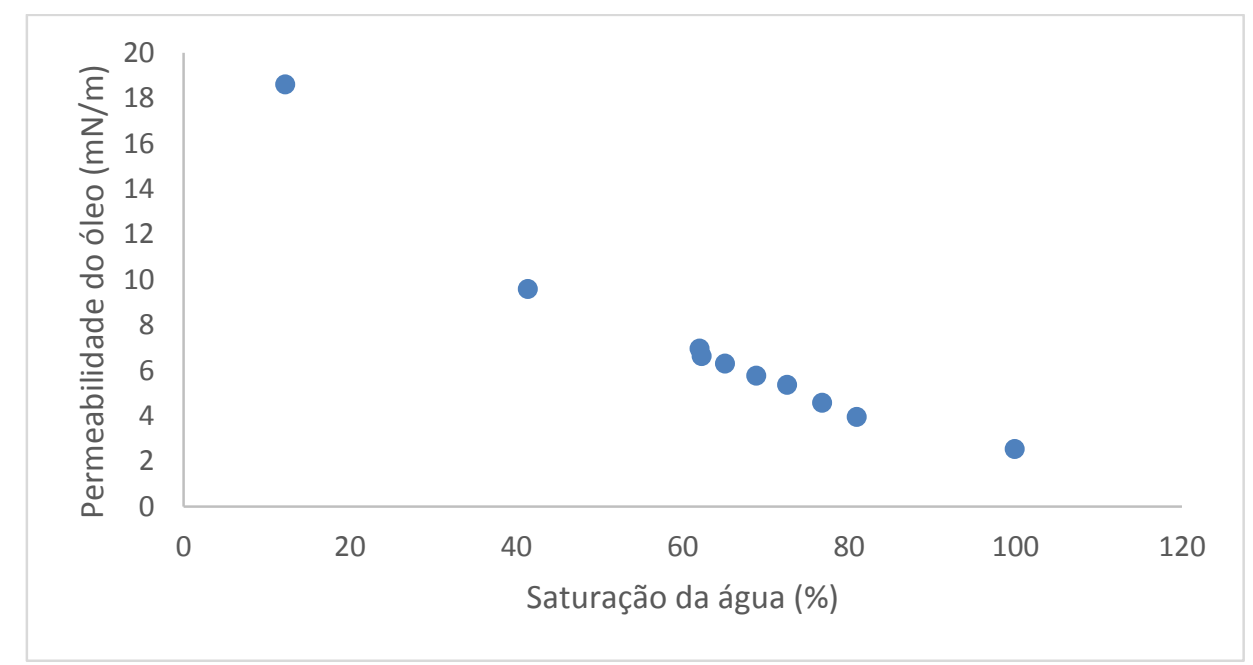

Figura 3: Permeabilidade absoluta para óleo

Na Figura 3 observamos que com aumento da permeabilidade do óleo ocorre a diminuição da saturação da água. Neste processo, como descrito na metodologia, o meio estava saturado com água e na medida que se adicionava óleo a saturação da água diminui e a permeabilidade do óleo aumenta, assim como acontece nos poços de petróleo.

\subsection{Fator de Recuperação Parcial e Total}

Nas análises do fator de recuperação parcial e total foram utilizadas as concentrações de biossurfactantes de $65 \times 10^{-6} \mathrm{~kg} / \mathrm{L}$ e $80 \times 10^{-6} \mathrm{~kg} / \mathrm{L}$. No início foi injetado a de $65 \times 10^{-6} \mathrm{~kg} / \mathrm{L} \mathrm{e}$ depois a concentração foi aumentando até $80 \times 10^{-6} \mathrm{~kg} / \mathrm{L}$ com objetivo de se obter uma maior recuperação do óleo.

No início o meio poroso foi saturado com $0,170 \mathrm{~L}$ de água e depois começou a passagem do óleo, ficando retido no meio poroso $0,070 \mathrm{~L}$ de óleo e $0,045 \mathrm{~L}$ de água (total $=0,115 \mathrm{~L}$ ). Em seguida visando reproduzir as condições do poço de petróleo, iniciou a passagem da salmoura e verificou que saiu 0,020 L de óleo. Logo, ficou 0,050 L de óleo, denominado de óleo residual, para ser recuperado pela injeção de biossurfactante, ou seja, simulando a MEOR como acontece nas indústrias de petróleo.

Na utilização do biossurfactante (caldo fermentado sem células) verificou-se que $0,015 \mathrm{~L}$ foram recuperados, obtendo-se segundo a Equação 01 um o fator de recuperação parcial (FRP) de 30,00 $\%$ referente somente a injeção do biossurfactante e um fator de recuperação total (FRT) de 50,00 $\%$ determinado com base na Equação 02, que representa a recuperação do óleo pela passagem da salmoura e do biossurfactante.

$$
F R P=\frac{\text { VOLUME DE ÓLEO RECUPERADO }(\text { ME })}{\text { VOLUME DE ÓLEO RESTANTE NO TESTEMUNHO }}=\frac{0,015}{0,050}=30,00 \%
$$


$F R T=\frac{\text { VOLUME TOTAL DE ÓLEO RECUPERADO }}{\text { VOLUME DE ÓLEO RESIDUAL }}=\frac{0,020+0,015}{0,070}=50,00 \%$

\section{CONCLUSÃO}

O biossurfactante produzido permaneceu estável frente à variação de temperatura e as altas concentrações salinas.

A concentração micelar crítica (CMC) para o caldo fermentado sem células contendo o biossurfactante foi de $50 \times 10^{-6} \mathrm{~kg} / \mathrm{L}$.

$\mathrm{Na}$ aplicação do biossurfactante (caldo fermentado sem células) no processo de recuperação do óleo residual resultou num fator de recuperação parcial de $30 \%$ a mais quando comparado com a utilização de apenas água e um fator de recuperação total de $50 \%$.

$\mathrm{O}$ aumento na eficiência de recuperação nos mostra o potencial deste bioproduto e contribui para uma viabilização econômica, visto que as recuperações de óleos residuais em poços são de alto custo.

\section{AGRADECIMENTOS}

Os autores agradecem aos órgãos financiadores FAPITEC, PETROBRAS, CAPES, CNPq e UFS pelo apoio financeiro através de bolsas e financiamento de projetos.

\section{REFERÊNCIAS BIBLIOGRÁFICAS}

1. Akbas H, Iscan M, Sidim T. Composition of Mixed Anionic/Nonionic Surfactant Micelles. J Surfactants Deterg. 2000 Jan; 3(1):77-80, doi: s1 1743-000-0117-0.

2. Al-Bahry SN, Al-Wahaibi YM, Elshafie AE, Al-Bemani AS, Joshi SJ, Al-Makhmari HS, Al-Sulaimani HS. Biosurfactant production by Bacillus subtilis B20 using date molasses and its possible application in enhanced oil recovery. Int Biodeter Biodegr. 2013 nov; 81:141-146, doi: 10.1016/j.ibiod.2012.01.006.

3. Ghojavand H, Vahabzadeh F, Shahraki AK. Enhanced oil recovery from low permeability dolomite cores using biosurfactant produced by a Bacillus mojavensis (PTCC 1696) isolated from Masjed-I Soleyman field. J Petrol Sci Eng. 2012, 81: 24-30, doi: 10.1016/j.petrol.2011.12.002

4. Barros FFC, Quadros CP de, Maróstica Júnior MR, Pastore GM. Surfactina: Propriedades Químicas, Tecnológicas e Funcionais para Aplicações em Alimentos. Quim Nova. 2007; 30 (2): 409-414, doi: 10.1590/S0100-40422007000200031.

5. Silva CF; Estudo da produção de biossurfactante utilizando resíduos agroindustriais. [Tese de Doutorado]. São Cristóvão, SE (Brasil); Universidade Federal de Sergipe; 2011. 163 p.

6. Albuquerque HS, Vale TYF, Dantas TN de C, Avelino de Neto A, Santanna VC, Coelho TAM. Estudo da eficiência de sistemas microemulsionados na recuperação avançada de petróleo. Anais do $4^{\circ}$ PDPETRO; 2007 Outubro; Campinas, São Paulo (Brasil).

7. Mulligan CN, Young RN, Gibbs BF. Heavy metal removal from sediments by biosurfactants. J Hazard Mater. 2001; 85:111-125, PMID: 11463506.

8. Wei YH, Chou LC, Chang JS. Rhamnolipid production by indigenous Pseudomonas aeruginosa J4 originating from petrochemical wastewater. Biochem Eng J. 2005; 27: 146-154, doi: 10.1016/j.bej.2005.08.028.

9. Gustavo WM. Análise entrópica de meios porosos utilizando os conceitos de entropia da informação de Shannon. [Monografia]. Vitória, ES (Brasil); Universidade Federal do Espirito Santo; 2007. 103 p. 